\title{
INTRODUCTION TO FOUNTAINS, WIND, INFALL (AND MAGNETIC FIELDS AND DYNAMO MECHANISMS)
}

\author{
F. D. KAHN \\ Department of Astronomy \\ The University \\ Manchester M13 9PL \\ England
}

\begin{abstract}
The galactic fountain is formed by hot gas rising from the galactic disk. The lines of force of the interstellar magnetic field are dragged along in the flow. This lecture deals with the geometry and topology of the configuration of the field that is set up. The fountain flow acts as a dynamo, and the presence of the magnetic field plays an important part in the ionization balance of the gas as it returns towards the disk.
\end{abstract}

\section{INTRODUCTION}

The galactic fountain is essentially a dynamic phenomenon. That is the key to understanding its properties. The motion is driven by the hot inter-cloud medium which itself derives its energy from supernova remnants. Models of this kind have been discussed before (Bregman, 1980; Kahn, 1981, 1989), but here the treatment is extended to include effects due to the galactic magnetic field. The lines of force in such a flow eventually detach themselves from the material that they are linked to in the disk. The excursion into the fountain eventually causes the field to be amplified and therefore constitutes a dynamo mechanism. The presence of the field also has a dominant effect on the pressure balance in gas clouds falling back towards the disk, and needs to be allowed for in the interpretation of relevant observations. In particular an infalling cloud will only be observed as neutral gas at $21 \mathrm{~cm}$ if it has a sufficiently large surface density.

\section{SUPERNOVAE, THE SOURCES OF THE FOUNTAIN FLOW}

A galactic fountain must be supported dynamically: it is very hard to argue for any alternative model, in which flow speeds are subsonic and support is essentially hydrostatic. The 
discussion here deals with dynamic fountains and will be based on a specification of the underlying galactic structure, as follows (Sellwood and Sanders, 1988):

$50 \%$ by mass of the Galaxy belongs to Population II, with its contents distributed so that the mass contained within radial distance $r$ of the centre is

$$
M(r)=\mu r
$$

$50 \%$ by mass belongs to Population I, with its contents distributed in a thin disk, so that the mass contained within distance $\varpi$ of the axis is

$$
M(\varpi)=\mu \varpi ;
$$

the rotation speed in the Galaxy is

$$
V=250 \mathrm{~km} \mathrm{~s}^{-1},
$$

the same at all axial distances, and then

$$
\mu=4.7 \times 10^{21} \mathrm{gm} \mathrm{cm}^{-1} .
$$

Adopting a value of $10 \mathrm{kpc}$ for the distance from the Sun to the galactic centre, the halfsurface density of the disk becomes

$$
\Sigma=\frac{\mu}{4 \pi \varpi}=1.24 \times 10^{-2} \mathrm{gm} \mathrm{cm}^{-2} .
$$

Just above the galactic disk the gravitational acceleration towards the disk, due almost entirely to Population I, is

$$
g_{z}=4 \pi G \Sigma=10^{-8} \mathrm{~cm} \mathrm{~s}^{-2} \text {. }
$$

The fountain rises to a height $Z=3 \mathrm{kpc}$ above the galactic plane, according to the generally accepted view. The galactic disk has a half-thickness much smaller than $Z$; in a quasi-static fountain the scale height of the distribution would have to be

$$
\frac{k T}{\bar{m} g_{z}} \sim Z
$$

where $T$ is the temperature and $\bar{m}\left(\sim 10^{-24} \mathrm{gm}\right)$ the mean particle mass, and therefore

$$
T \sim 7 \times 10^{5} \mathrm{~K} .
$$

The dynamical relaxation time of such a structure is

$$
t_{\mathrm{dyn}}=\left(\frac{k T}{\bar{m}}\right)^{\frac{1}{2}} / g_{z}=10^{15} \mathrm{~s}=30 m y ;
$$

if the fountain can be set up by subsonic motions, $t_{\text {dyn }}$ must be short compared with

$$
t_{\text {therm }}=3 k T / \Lambda(T) n,
$$

the thermal relaxation time. Here

$$
\Lambda(T) \sim \lambda T^{-\frac{1}{2}}
$$


is the usual cooling function, and $\lambda=10^{-19}$ in CGS units, while $n$ is the atom/ion density (Kahn, 1976). The condition implies that the column density in the halo is limited by the inequality

$$
n Z \ll 3\left(\frac{\bar{m}^{3}}{k}\right)^{\frac{1}{2}} \frac{g^{2} Z^{2}}{\lambda}=2.5 \times 10^{19} \mathrm{~cm}^{-2} .
$$

Only a very tenuous fountain would obey this restriction. So a quasi-static model of the fountain proves to be unsatisfactory, and it clearly is necessary to consider dynamical models.

Three distinct possibilities of this kind have been proposed:

(i) The fountain flow consists of gas coming from the innermost parts of many supernova remnants. The essential point here is that the inner parts of a remnant are heated very strongly by the blast wave from the explosion, and take a very long time to lose their thermal energy by radiation. The flow into the fountain is therefore thought to consist of the amalgamated hot remains of many neighbouring SNR's.

(ii) A popular alternative view is that the major contribution to the flow comes from the combined remnants of many supernovae that have occurred close together, in space and time, at the sites of evolving OB associations. There is enough energy in such a combined explosion to create a blast that breaks right through the galactic disk, and ejects matter ballistically into the space above. The driving force is still provided by hot gas (Norman, 1990).

(iii) The third view stresses the possible importance of cosmic rays in driving the flow. Once more the fountain is spread more or less homogeneously over the galactic disk. The internal energy that sets up the motion is provided by the cosmic rays; they are coupled to the thermal plasma by Alfvén waves, and drag it along as they escape from the galactic disk (Völk, 1990).

Both (ii) and (iii) are dealt with in later papers at this Symposium. Here I shall explore the possibilities offered and the restrictions imposed by the first mechanism.

The first question to ask concerns the spacing of supernova remnants. If one waits long enough, then neighbouring supernova remnants will merge. By the time this happens a particular remnant will be well into Phase III. The Sedov solution predicts that the radius of a remnant is

$$
r=a t^{\frac{2}{5}} \equiv\left(\frac{2 E}{\bar{\rho}}\right)^{\frac{1}{5}} t^{\frac{2}{5}}
$$

during Phase II; here the energy of the supernova explosion is $E\left(=3 \times 10^{51} \mathrm{erg}\right)$ and the density of the surrounding medium is $\bar{\rho}\left(=2 \times 10^{-24} \mathrm{gm} \mathrm{cm}^{-3}\right)$. Phase II comes to an end when radiative energy loss becomes important, at time

$$
t=t_{*}=1.1 \frac{E^{\frac{3}{14}}}{q^{\frac{5}{14}} \rho^{\frac{4}{7}}} .
$$

The term $q$ here is a coefficient introduced in a simplified cooling law suitable for interstellar gas dynamics, in the temperature range $3 \times 10^{5} \mathrm{~K}<T<3 \times 10^{7} \mathrm{~K}$, see $\mathrm{Kahn}$ (1976). Its numerical value is $4 \times 10^{32}$, in CGS units, for a gas mixture with solar abundances.

Before time $t_{*}$ the expansion of the SNR is driven mainly by gas pressure, and after 
time $t_{*}$ it becomes momentum driven; the radius at time $t$ is then given by

$$
r=b t^{\frac{1}{4}} \text {. }
$$

The coefficient here can be expressed in terms of $E, q$ and $\bar{\rho}$ by the relation

$$
b=0.92 \frac{E^{\frac{13}{56}}}{q^{\frac{3}{56}} \bar{\rho}^{\frac{2}{7}}},
$$

with a very weak dependence on $q$, and therefore on the precise form of the cooling law.

Supernova explosions of type II seem to occur about three times per century per galaxy, effectively in a volume

$$
\begin{aligned}
\mathcal{V} & =\pi \times(10 \mathrm{kpc})^{2} \times 200 \mathrm{pc} \\
& =1.7 \times 10^{66} \mathrm{~cm}^{3}
\end{aligned}
$$

and their rate of occurrence, per unit volume and time, is

$$
\nu=6 \times 10^{-76} \mathrm{~cm}^{-3} \mathrm{~s}^{-1} \text {. }
$$

Now imagine that the Galaxy had started at time zero entirely free of supernova remnants, and that SNR's were subsequently created at rate $\nu$ and allowed to expand without interference from each other. The available space in the disk would be filled when

$$
\frac{4 \pi}{3} b^{3} t^{\frac{3}{4}} \nu t=1
$$

or

$$
t=\left(\frac{3}{4 \pi}\right)^{\frac{4}{7}} b^{-\frac{12}{7}} \nu^{-\frac{4}{7}}=3.9 \times 10^{13} \mathrm{~s}=1.2 \text { million years; }
$$

the radius of the typical remnant would be

$$
r=2.2 \times 10^{20} \mathrm{~cm} \sim 70 \mathrm{pc}
$$

then. These estimates compare well with the linear dimensions and inferred ages of the known loop structures in the neighbourhood of the Sun.

Even at this late stage a SNR still contains shock-heated gas which has not yet lost its thermal energy. The effect of radiative losses is conveniently described by the equation (Kahn 1976)

$$
\frac{D}{D t} \kappa^{\frac{3}{2}}=-q
$$

where $D / D t$ denotes rate of change following the motion of a fluid element, and

$$
\kappa \equiv \frac{P}{\rho^{\frac{5}{3}}}
$$

is the adiabatic parameter. Equation (17) applies only while the gas still retains some heat; a particular fluid element has cooled off completely, and occupies only a small volume, when 
$\kappa$ reaches zero. If a fluid element is to stay hot for time $t_{h}=10^{6}$ years, then it must acquire so much thermal energy from the blast wave that

$$
\kappa^{\frac{3}{2}}=\kappa_{s}^{\frac{3}{2}}>q t_{h}=1.2 \times 10^{46}
$$

at the time that the blast wave passes across it. Each supernova explosion produces about $200 M_{\odot}$ of interstellar gas which are shock-heated to this extent. On the present model this gas is still hot when neighbouring SNR's merge with one another, and is available for flow into the fountain. A supernova rate of three per century produces a flow of gas into the fountain at the rate of $6 M_{\odot}$ per year. This estimate agrees well with the mass infall rate from the halo deduced by Wakker (1990a). The total mass of interstellar gas in the disk is

$$
\begin{aligned}
M_{i s} & =2 \times 10^{-24} \times 1.7 \times 10^{66} \mathrm{gm} \\
& =1.7 \times 10^{9} M_{\odot},
\end{aligned}
$$

with the present figures, so that the typical interstellar atom will typically make an excursion into the fountain once every 300 million years.

The flow takes place on each side of the galactic disk over an area some $10 \mathrm{kpc}$ in radius; the mass flux is therefore

$$
\begin{aligned}
\Phi & =\frac{6}{6 \times 10^{8}} M_{\odot} \mathrm{pc}^{-2} \mathrm{yr}^{-1} \\
& =7 \times 10^{-20} \mathrm{gm} \mathrm{cm}^{-2} \mathrm{~s}^{-1}
\end{aligned}
$$

The fountain has to rise to a maximum height $Z=3 \mathrm{kpc}$, and so requires enthalpy

$$
\mathcal{E}=g_{z} Z=10^{14} \mathrm{erg} \mathrm{gm}^{-1} .
$$

In terms of $P, \rho$ and $\kappa$, then,

$$
\frac{2}{3} \frac{P}{\rho}=\mathcal{E}
$$

and if the adiabatic parameter given by

$$
\kappa^{\frac{3}{2}} \sim 10^{46} \text { (CGS units) }
$$

is taken as typical, then

$$
\begin{aligned}
P & =\left(\frac{2}{3} \mathcal{E}\right)^{\frac{5}{2}} \kappa^{-\frac{3}{2}} \\
& =3.6 \times 10^{-12} \text { dyne } \mathrm{cm}^{-2}
\end{aligned}
$$

This value is somewhat larger than the interstellar pressure. A more careful discussion is needed for a more accurate determination of the parameter $\kappa$.

The upward flow leaves the disk with speed $u_{0}$, and produces a recoil force, per unit area,

$$
\Phi u_{0}=10^{-12} \text { dyne } \mathrm{cm}^{-2},
$$


which agrees well with the usual value for the interstellar pressure.

If the fountain were purely one-dimensional there would be no need to consider any possible sideways drift parallel to the galactic plane. But the maximum height is comparable to typical distances within the disk, say that to the galactic centre. It is therefore relevant to note that the component of the gravitational acceleration in the direction towards the axis of symmetry is

$$
g_{\varpi}=\frac{V^{2}}{\varpi_{0}}-\frac{V^{2}}{\varpi_{0}^{2}}\left(\Pi+\frac{|z|}{2}\right)+\ldots,
$$

appropriate to the mass distribution assumed earlier. The symbols here mean that the fluid element has been displaced a distance $z$ vertically and a distance $\Pi$ axially from its original position in the plane, at axial distance $\varpi_{0}$. The material undergoes a Lindblad oscillation, whether or not it is in the galactic disk. The displacement $|z|$ away from the plane reduces the centripetal acceleration and causes the gas to drift outwards, and therefore also to lag in its rotation about the galactic axis. There is not space here to discuss the full implications of this effect, except to remark that the gas returning from the fountain will not, in general, fall back onto the galactic disk at exactly the same axial distance as that where it originated. Mixing is therefore promoted by the fountain flow, as well as transport of angular momentum, and cross-linkages in the galactic magnetic field.

\section{AMPLIFICATION OF THE MAGNETIC FIELD.}

The flow into the fountain consists of the combined parts of remnants from many supernova explosions, closely packed together like a set of bubbles in a foam. It can be described neither in terms of a single large bubble expelled by itself from the disk, nor in terms of a smooth continuum flow, since the individual bubbles themselves have diameters comparable with the thickness of the disk.

An important consequence of this model is that it naturally leads to the amplification of the galactic magnetic field. The $200 M_{\odot}$ of hot gas which fill the bubble when it amalgamates with its neighbours were originally contained in a spherical volume of radius $r_{0}=$ $12 \mathrm{pc}$, and then expanded to a radius $r_{f}=70 \mathrm{pc}$, an increase in linear dimensions by a factor of about 6 . The corresponding density decrease is by a factor 200 or so, and the flow upwards starts with a density $\rho_{f} \sim 10^{-26} \mathrm{gm} \mathrm{cm}^{-3}$. The magnetic field linking the original distribution of material has strength $B_{0} \sim 3 \times 10^{-6}$ gauss. The magnetic flux linked to the bubble is

$$
\Phi_{\text {mag }}=\pi B_{0} r_{0}^{2}=1.22 \times 10^{36} \text { gauss cm }{ }^{2}
$$

and remains conserved during the three-dimensional expansion. When the bubble reaches radius $r_{f}$, the horizontal field strength has therefore dropped to $B_{f} \sim 10^{-7}$ gauss, and the ratio $B / \rho$ has increased by a factor 6 , from $1.5 \times 10^{18}$ gauss $\mathrm{cm}^{3} \mathrm{gm}^{-1}$ to $9 \times 10^{18}$ gauss $\mathrm{cm}^{3} \mathrm{gm}^{-1}$. Even after the expansion each bubble stays magnetically linked to the same elements of the gas as before the supernova explosion, all of them lying further out in the SNR. Consequently these elements will have been heated to a much smaller extent by the blast wave, and can cool off again by the time that the bubble drifts off into the fountain. So each rising bubble still has tubes of force trailing from it, connecting it to 
interstellar matter that remains behind in the disk. A complex magnetic pattern results in the fountain, with a systematic field component parallel to the original lines of force in the disk, as well as a vertical component, perpendicular to the disk, whose net flux is zero, but whose average field strength becomes dominant in the lower part of the flow.

If there were no reconnection of field lines the vertical component would eventually become so strong as to dominate flow entirely. Let the typical bubble have linear dimensions $\ell$ and contain horizontal magnetic field $B_{\|}$, linked to the galactic disk below. The associated vertical field $B_{\perp}$ then has total flux $B_{\|} \ell$ per unit width going up on one side of the bubble, and equal flux going down on the other. When the fountain has been flowing for some time, and there are $n$ layers of bubbles stacked on top of one another, then tubes of force with an upward flux, per unit width, of $n B_{\|} \ell$, as well as an equal downward flux, have to be fitted into a length $\ell$. The resulting vertical field averages to zero, and has typical magnitude

$$
\left|B_{\perp}\right|=2 n B_{\|}=\frac{2 n B_{0}}{f^{2}} .
$$

The spacing between vertical lines of force with opposite directions is typically $\ell / n$. The Petschek mechanism (Petschek, 1964; Baum and Bratenahl, 1977) will cause reconnection in this field at the typical rate

$$
\sigma \sim \frac{0.1 B_{\perp} n}{\sqrt{4 \pi \rho} \ell}
$$

New vertical field is created by the flow at rate

$$
\left(\frac{d B_{\perp}}{d t}\right)_{\text {new }}=2 B_{\|} \frac{v}{\ell}=\frac{B_{\perp} v}{n \ell}
$$

reconnection halts the increase when

$$
\sigma \equiv \frac{0.1 B_{\perp} n}{\sqrt{4 \pi \rho} \ell}=\frac{v}{n \ell}
$$

or

or

$$
n^{2}=\frac{10 \sqrt{4 \pi \rho} v}{B_{\perp}}
$$

$$
n^{3}=\frac{5 \sqrt{4 \pi \rho} v}{B_{\|}} .
$$

With the values adopted here, it is found that $n \sim 6$, and then setting $\ell=140 \mathrm{pc}$, the typical diameter of a bubble, it follows that the vertical magnetic field should extend to about $840 \mathrm{pc}$ height; $\left|B_{\perp}\right|$ typically equals $1.2 \times 10^{-6}$ gauss.

The vertical field will disappear at heights greater than about $1 \mathrm{kpc}$, and only a connected $B_{\|}$field will remain. The later upward flow is essentially one-dimensional, and so is the final collapse back to the galactic plane. The gas that returns from the fountain has a value of $B_{\|} / \rho$ that is some six times larger than it was before it was expanded by the supernova remnant. The interstellar gas makes excursions into the fountain typically at intervals of $\mathbf{3 0 0}$ million years. On the face of it the magnetic field strength in the disk should increase by a factor 6 once every 300 million years, and so should double every 120 million years, but such a rate of increase is impossible to sustain. 
In fact there are significant restrictions which limit the possible amplification rate. The enhanced magnetic field $B_{\|}$can only be set up if each bubble in the fountain reconnects magnetically with other bubbles on either side. The linkage will be broken in places where there is no fountain flow. The amplification process will thus only produce extended magnetic loops lying above regions of the galactic disk with an active fountain flow.

A second limit is set by the conditions that the magnetic pressure term $B_{\perp}^{2} / 8 \pi$ must be significantly smaller than $\rho v^{2}\left(\sim P_{i s}\right)$, the momentum flow into the fountain. Formally the restriction is that

$$
\frac{B_{\perp}^{2}}{8 \pi}=\frac{n^{2} B_{0}^{2}}{2 \pi f^{4}} \ll P_{i s}
$$

the interstellar pressure, and writing relation (27) in the form

$$
\frac{n^{3} B_{0}}{f^{2}}=5 \sqrt{4 \pi P_{i s}}
$$

one finds, after a simple calculation, that

$$
\frac{B_{0}^{2}}{4 \pi P_{i s}} \ll \frac{f^{4}}{5 \times 2^{3 / 2}}
$$

so that $B_{0}$ must be considerably smaller than $3 \times 10^{-5}$ gauss, with $f=6$. The amplification begins to fail when the galactic magnetic field is rather larger than $10 \mu G$.

A lower limit is set on $B_{0}$ by the requirement that reconnection must occur fast enough, or else the entire galactic field might be swept into the fountain, leaving nothing behind to be amplified. The flux per unit width in the disk is $B_{0} D$ and this must always be rather larger than $B_{\perp} \ell / 2$, which is the flux still connected to bubbles in the fountain. So

or

$$
B_{0} D \gg \frac{1}{2} B_{\perp} \ell=n B_{\|} \ell=\frac{n B_{0} \ell}{f^{2}}
$$

$$
\frac{n}{f^{2}} \ll \frac{D}{\ell}
$$

and then with the help of relation (27) and a little manipulation

$$
\frac{B_{0}^{2}}{4 \pi P_{i}} \gg \frac{25 \ell^{6}}{f^{8} D^{6}} \text {. }
$$

With our values the implied minimum field strength is $1.3 \times 10^{-8}$ gauss.

To sum up, this process will rearrange the magnetic field and create closed lines of force above regions in the galactic disk that have a fountain flow. All the interstellar gas is cycled through the fountain at intervals of a few hundred million years. The amplification process described here systematically increases the ratio $B_{0}: \rho_{0}$; it operates provided that

$$
10^{-8} \text { gauss }<B_{0}<10^{-5} \text { gauss } \text {. }
$$

\section{DESCENDING GAS IN THE FOUNTAIN: INFLUENCE OF THE MAGNETIC FIELD}

The fountain flow plays an important role in maintaining the magnetic field of the Galaxy, as was shown in the previous section. Now I shall discuss the influence of the magnetic field 
on the structure and ionization balance of the gas as it descends towards the galactic plane. The flow is essentially one-dimensional, so that the horizontal field strength is related to the density by the formula

$$
B_{\|}=K \rho
$$

where typically $K=10^{19}$ gauss $\mathrm{cm}^{3} \mathrm{gm}^{-1}$. There is also the vertical component $B_{\perp}$ which has greater field strength at low altitudes but contributes no net flux, and can be ignored above about $1 \mathrm{kpc}$ height. For this simple calculation it seems adequate to treat the motion as one-dimensional.Let the upward speed of flow be $u$ at height $z$ (greater than $1 \mathrm{kpc}$ ). Let there be a cooled sheet of gas at this height, with surface density $\Sigma$, descending at speed $w$. Two conditions specify its motion and its evolution: they are

conservation of mass

$$
\frac{d}{d t} \Sigma w=\frac{\Phi}{u}(u+w)
$$

and conservation of momentum

$$
\frac{d}{d t}(\Sigma w)=\Sigma g-\Phi(u+w) .
$$

The cooled sheet sets itself internally like an atmosphere under an effective gravitational acceleration

$$
g_{\mathrm{eff}}=g-\frac{d w}{d t}=\frac{\Phi}{\Sigma u}(u+w)^{2} ;
$$

in the simplest case it consists only of matter that has reached maximum height in the fountain, and is now falling back. In that case

$$
w=\frac{u}{2}
$$

and

$$
\Sigma=3 \Phi \frac{u}{g}
$$

and so

$$
g_{\mathrm{eff}}=\frac{3 g}{4} .
$$

But the falling sheet may be pushed from above by extragalactic matter that has entered from outside. In that case $w$ will differ from the value given in (37), say

$$
w=W u / 2,
$$

and the effective gravitational acceleration in the layer changes to

$$
g_{\mathrm{eff}}=\frac{g}{3}\left(1+\frac{W}{2}\right)^{2} .
$$

Suppose now that $P_{\text {eff }}$ is the effective pressure (including magnetic effects) at the base of the descending layer, and let $\rho_{b}$ be the gas density there, then

$$
\begin{aligned}
P_{\mathrm{eff}} & =\frac{B^{2}}{8 \pi}+\rho_{b} c_{i}^{2} \\
& =\frac{K^{2} \rho_{b}^{2}}{8 \pi}+\rho_{b} c_{i}^{2},
\end{aligned}
$$


and here $c_{i}\left(\equiv 10 \mathrm{~km} \mathrm{~s}^{-1}\right)$ is the isothermal sound speed in the gas, assumed to be ionized. The momentum balance at the base of the sheet requires that

$$
P_{\mathrm{eff}}=\Phi u\left(1+\frac{W}{2}\right)^{2} \text {. }
$$

Magnetic pressure dominates whenever

$$
\rho_{b}>\frac{8 \pi c_{i}^{2}}{K^{2}}=2.5 \times 10^{-25} \mathrm{gm} \mathrm{cm}^{-3},
$$

with the values of the parameters being used here, and therefore also if

$$
P_{\text {eff }}>\frac{16 \pi c_{i}^{4}}{K^{2}}=5 \times 10^{-13}{\text { dyne } \mathrm{cm}^{-2}}
$$

or

$$
u>\frac{P_{\mathrm{eff}}}{\Phi\left(\left(1+\frac{W}{2}\right)^{2}\right.}=\frac{7 \times 10^{6}}{\left(1+\frac{W}{2}\right)^{2}} \mathrm{~cm} \mathrm{~s}^{-1} .
$$

$W$ has to exceed unity, and so the least favourable value of the limiting speed on the right hand side is $30 \mathrm{~km} \mathrm{~s}^{-1}$. The description from now on is confined to heights in the fountain greater than $1 \mathrm{kpc}$, so that the $B_{\perp}$ component of the field is absent, and more than 150 pc below the highest level in the flow, so that $u$ exceeds $30 \mathrm{~km} \mathrm{~s}^{-1}$, and magnetic pressure dominates. The condition of hydrostatic equilibrium in the descending sheet then states that

or

$$
\frac{d P_{\mathrm{eff}}}{d z}=\frac{K^{2} \rho}{4 \pi} \frac{d \rho}{d z}=-g_{\mathrm{eff}} \rho
$$

$$
d z=-\frac{K^{2}}{4 \pi g_{\mathrm{eff}}} d \rho
$$

Let $F_{\text {ion }}$ be the flux of Lyman continuum photons coming up into the fountain from unshielded early-type stars in the galactic disk. If $F_{\text {ion }}$ is large enough, or the surface density $\Sigma$ small enough, then the sheet will be turned entirely into an HII region. In such a case no high (or intermediate) velocity cloud will be detected at $21 \mathrm{~cm}$ wavelength.

The value of $F_{\text {ion }}$ defines a minimum surface density required before neutral hydrogen will be found in the sheet. The necessary condition is that

$$
F_{\text {ion }}<\int b n_{e}^{2} d z
$$

where $b$ is the usual recombination coefficient for hydrogen, $n_{e}$ is the electron density and the integration extends through the sheet. The electron density in the ionized gas is related to the mass density by

$$
n_{e}=\rho / m_{a}
$$

where $m_{a}\left(=2 \times 10^{-24} \mathrm{gm}\right)$ equals the mass of atoms/ions present per free electron. With the help of (45) and (47) the inequality becomes

$$
\begin{aligned}
F_{\text {ion }} & <\frac{b K^{2} \rho_{b}^{3}}{12 \pi g_{\text {eff }} m_{a}^{2}} \\
& =\frac{1.92 b \Sigma^{3 / 2} g^{1 / 2}(1+W / 2)}{m_{a}^{2} K}
\end{aligned}
$$


after some reduction. The table below summarises the result. The calculation assumes that $W=1$, in other words that the descending sheet is not pushed from behind by the infall of extragalactic material. But relation (48) shows that it is quite easy to allow for such an infall, and that the correction will not make a qualitative difference to the conclusions.

\begin{tabular}{l|cccc}
$\begin{array}{l}\text { Mass per unit } \\
\text { area } \Sigma\end{array}$ & $10^{-5}$ & $10^{-4}$ & $10^{-3}$ & $\mathrm{gm} / \mathrm{cm}^{2}$ \\
\hline $\begin{array}{l}\text { Upper limit to the } \\
\text { ionizing flux from } \\
\text { below } F_{\text {ion, max }}\end{array}$ & $4.6 \times 10^{4}$ & $1.4 \times 10^{6}$ & $4.6 \times 10^{7}$ & photon $/ \mathrm{cm}^{2} \mathrm{~s}$
\end{tabular}

The most likely suitable value for $F_{\text {ion, } \max }$ is of order $10^{6}$ photon $/ \mathrm{cm}^{2} \mathrm{~s}$ (Bregman and Harrington, 1986; Reynolds, 1989). The results quoted in the table show that a Lyman c flux as strong as this will maintain full ionization in an infalling cloud unless the mass per unit area is at least of order $10^{-4} \mathrm{gm} \mathrm{cm}^{-2}$, or the surface density of particles at least of order $10^{20} \mathrm{~cm}^{-2}$. It is relevant therefore that the typical surface density of $\mathrm{H}$ atoms in an HVC is also of this order (Wakker, 1990b). Any interpretation of the HVC's must therefore make allowance for the inflow of the ionized part of a cloud that necessarily accompanies it.

\section{REFERENCES}

Baum, P. J., Bratenahl, A. (1977) J. Plasma Phys. 18, 257.

Bregman, J. N. (1980) Ap. J. 236, 577.

Bregman, J. N., Harrington, J. P. (1986) Ap. J. 309, 833.

Kahn, F. D. (1976) Astron. Astrophys. 50, 145.

Kahn, F. D. (1981) "Dynamics of the Galactic Fountain" in Investigating the Universe, ed. F. D.Kahn, Reidel, Dordrecht, p. 1.

Kahn, F. D. (1989) "Galactic Fountains" in Structure and Dynamics of the Interstellar Medium, IAU Colloquium 120, eds. G. Tenorio-Tagle, M. Moles, J. Melnick, Springer, p. 474.

Norman, C. (1990) Invited lecture at this Symposium.

Petschek, H. E. (1964) NASA SP-50, p. 425.

Reynolds, R. J. (1989) Ap. J. 339, L29.

Völk, H. J. (1990) Invited lecture at this Symposium.

Wakker, B. P. (1990a) Ph.D.thesis, Rijksuniversiteit Groningen.

Wakker, B. P. (1990b) Invited lecture at this Symposium. 